\title{
Gerhard Ebeling
}

Rückwendung zur Reformation und Wortgeschehen*

Paul Ricœur

Unser Problem ist das der Theorie des Wortes, das übrigens den Ausdruck Theologie des Wortes umso mehr rechtfertigt, als der Begriff des Wortes in seinem Zusammenhang und als Fundamentalkonzept verstanden wird.

Ich werde mich also an Ebeling orientieren, der in Frankreich kaum bekannt ist, in Deutschland hingegen, im Kontext der neuen Wahrnehmung des hermeneutischen Problems, die dominante Figur in Kreis derer darstellt, die man schon - und vielleicht etwas voreilig - die "Post-Bultmannianer" nennt.

Ebeling hat seit etwa 12 Jahren, seit 1954, eine Reihe von Arbeiten publiziert, und ich glaube, es ist wichtig, ihn zunächst in Bezug auf sein Arbeitsfeld zu situieren. Bultmann war Exeget, Ebeling ist Kirchenhistoriker. Es ist sehr wichtig zu sehen, dass diese Fachzugehörigkeit sein Problem bestimmt. Tatsächlich war es im Zuge seiner Beschäftigung mit Luther und der Reformation, dass ihm bewusst wurde: Die Geschichte der Kirche ist zunächst nicht eine Geschichte der Dogmen - sie ist eine Geschichte der Predigt, sie ist die Geschichte der Auslegung der Schrift ${ }^{1}$, wie sie in der Predigt ge-

* Erschienen unter dem Titel Ebeling, in: Foi-éducation, Nr. 37 (Okt.-Dez. 1967), S. 36-57 (Ricœur-Bibliographie, ed.Vansina, Nr. II.A.218). Übersetzung aus dem Französischen von Andreas Mauz. Es handelt sich um die schriftliche Übertragung eines Vortrags, die noch starke Züge der mündlichen Rede trägt. Auf die Übersetzung der Diskussion, die sich an den Vortrag anschloss (S. 5357), wird verzichtet. Anmerkungen und Nachweise stammen ausschliesslich vom Übersetzer, ebenso der Untertitel. In einigen Fällen schien es angezeigt, die Begrifflichkeit Ricœurs in eckigen Klammern nachzutragen. - Man vergleiche ferner Ricœurs Beiträge: L'événement de la parole chez Ebeling, in: Cahiers du Centre Protestant de l'Ouest, Nr. 9/1968, S. 23-31; Contribution d'une réflexion sur le langage à une théologie de la parole, in: RThPh 18 (1968), S. 333-348 [auch in: Xavier Léon-Dufour (Hg.), Exégèse et herméneutique, Paris 1971, S. 201-220; dt. Üs.: Sprache und Theologie des Wortes, in: Xavier Léon-Dufour (Hg.), Exegese im Methodenkonflikt, München 1973, S. 201-221].

${ }^{1}$ Anspielung auf Ebelings Tübinger Antrittsvorlesung von 1946, vgl. Gerhard Ebeling, Kirchengeschichte als Geschichte der Auslegung der Heiligen Schrift, Tübingen 1947 (SgV 189); erneut abgedruckt in: ders., Wort Gottes und Tradition. Studien zu einer Hermeneutik der Konfessionen, Göttingen 1964 (= Kirche und Konfession, Bd. 7), S. 9-27. 
schieht. Nicht in der exegetischen Arbeit also, sondern imVerstehen des Schicksals der Kirche und ihrer Predigt, stellt sich das zentrale Problem der Hermeneutik nach Bultmann ein.

An diese fachliche Differenz - ein Theologe in erster Linie vertraut mit der Kirchen- und Dogmengeschichte - schliessen sich weitere grundlegende Differenzen an, die man zur Kenntnis nehmen muss, bevor man sich auf Ebelings Werk einlässt. Zunächst ist es das Problem der Sprache, das ganz in den Vordergrund tritt, während es für Bultmann in gewisser Hinsicht ein Sekundärproblem darstellt, nachgeordnet nämlich dem Problem, das wir das der richtigen Begrifflichkeit [la juste conceptualité] genannt haben. ${ }^{2}$ Die generellen Bedingungen der Sprache und des Wortes treten ganz in den Vordergrund, und im gleichen Zug wird das Problem der Predigt in direkte Verbindung gebracht mit der Entwicklung unserer Kultur, die ihrerseits als Sprache verstanden wird, als ein Ensemble von Sprachspielen, wie man mit Wittgenstein ${ }^{3}$ sagen könnte. Das heisst: Unsere Kultur ist stets ein Wettbewerb innerhalb eines Ensembles von Sprachen, Techniken (kulturellen und anderen); und so, als eine Sprache unter anderen, kann die Predigt Einfluss nehmen auf die Kultur. Es handelt sich also darum, das Problem der Sprache nicht als das Sekundärproblem einer Ausdruckshülle in Bezug auf einen bereits ausgedrückten Inhalt aufzufassen, sondern als die grundlegende Übung des Zeugnisses der Kirche in einer Kultur, die ihrerseits als ein Ensemble von Sprachen verstanden wird.

Damit ist bereits ein erster Übergang im Kontext unseres Problems vollzogen. Es müssen nun noch zwei oder drei weitere ergänzt werden, bevor wir uns dem Werk Ebelings zuwenden. Zunächst ist da eine gewisse Verschiebung bezüglich der philosophischen Referenzen. Bultmann ist ganz und gar der Theologe der existenziellen oder existenzialistischen Periode der Theologie. Man kann nun nicht sagen, dass diese beendet sei, doch ist sie nicht mehr so dominant wie zur Zeit des frühen Heidegger; und die Mehrheit der Theologen, auf die ich mich beziehen werde (wobei ich Ebeling für den bemerkenswertesten unter ihnen halte), sind sehr viel empfänglicher für das späte Werk Heideggers als für das frühe. (Ich werde, wenn Sie erlauben, von Heidegger I und Heidegger II sprechen.) Heidegger I war massgeblich beschäftigt mit dem Problem der Kategorien der menschlichen Existenz: Was heisst es, dass wir als ein Wesen existieren, das geboren wird, das stirbt, das spricht? etc. - eine Problematik also, die wir eine existentiale genannt haben.

\footnotetext{
${ }^{2}$ Vgl. Ricœurs Beitrag über Bultmann im selben Heft, S. 17-35.

3 Im Orig. irrtümlicherweise »Vickenstein« (S. 37).
} 
In seinem späten Werk hat Heidegger eine recht andere Problematik angesprochen, die Frage nämlich nach dem Sinn des Wortes, verstanden als unabhängig von seinen subjektiven Bedingtheiten. Das ist, wenn Sie wollen, eine gewisse Rücknahme des Subjektivismus der Auslegung [interprétation] ${ }^{4}$ und der Zugang auf etwas, was man die Problematik des Sinns nennen könnte. Wenn ein Text spricht, ist dies nicht ein psychologisches Problem der Kommunikation von einem Menschen zum anderen; vielmehr gibt es einen Sinn, der sich zuvor des Textes bemächtigt und sich an jemanden wendet. Und diese Konstitution des Sinns in einem Werk ist prioritär. Es handelt sich also um eine Rücknahme des existenziellen Problems zugunsten des Problems des objektiven Sinns, eine Rückkehr, sagen wir, zu einem gewissen Objektivismus [objectivisme] des Sinns.

Ich möchte noch zwei andere Aspekte betonen, welche diese Periode prägen. Also: Unterordnung des partikulären Problems der Exegese unter die generelle Frage der Sprache, und innerhalb dieser Frage der Sprache Unterordnung des Problems des existenziellen Verstehens unter die Objektivität [objectivité] des Sinns. Anfügen möchte ich nun: ein Ernstnehmen des Problems der Säkularisation. Dies ist ein Problem, das Bultmann nicht besonders beschäftigt hat, da er, als Exeget, sehr viel stärker besorgt war um die mythologische Hülle des Textes. Die Mythologie ist, wenn Sie wollen, ein Problem des Exegeten. Doch für einen Kirchenhistoriker gibt es ein Problem, das sehr viel wichtiger ist: die Entwicklung unserer Kultur in Richtung einer globalen Säkularisation. Und hier hat sich nun eine Verbindung ergeben zwischen postbultmannscher Hermeneutik und Bonhoeffer-Einfluss. (Bonhoeffer, dieser junge Theologie, Barth-Schüler, der, wie Sie wissen, $1945^{6}$ nach mehreren Monaten Haft von Hitler exekutiert wurde.) In seinen Briefen aus dem Gefängnis hat er ein ausserordentliches und beunruhigendes Zeugnis hinterlassen, und hier spricht er eben zum erstenmal mit grossem Nachdruck von der neuen Situation des Christentums in einer gänzlich a-religiösen Welt. ${ }^{7}$ Dieses Problem eines nicht-religiösen Christentums ist es nun, das die existenzielle

\footnotetext{
4 "Interprétation" wird - Ricœur folgend (vgl. S. 42 bzw. S. 83 dieser Übersetzung), der seinerseits wohl Ebeling folgt - als "Auslegung" wiedergegeben; die wenigen Ausnahmen, in denen durch »Interpretation« übersetzt wird, beziehen sich auf Bonhoeffers Überlegungen zu einer "nicht-religiösen Interpretation biblischer Begriffe» (vgl. S. 51f. bzw. S. 92f. dieser Übersetzung).

5 Im Orig. konsequent »Bonhöffer« (vgl. S. 38ff.).

6 Im Orig. irrtümlicherweise "1944« (S. 38).

${ }^{7}$ Vgl. Dietrich Bonhoeffer, Widerstand und Ergebung. Briefe und Aufzeichnungen aus der Haft, hrsg. von Eberhard Bethge, München 1951.
} 
Note mit hereingebracht hat, die nicht mehr auf seiten der Philosophie zu suchen war, sondern auf jener der grundlegenden Bewegungen der Kultur. Folgedessen ist der Ort des Atheismus in der Theologie zu einer der dominierenden Fragen der Post-Bultmannianer geworden.

Schliesslich will ich ein letztes Moment anfügen. Da ich das Problem nicht gut kenne, werde ich mich kurz halten. Auf dem Feld der eigentlichen Exegese können wir seit einigen Jahrzehnten eine gewisse Entwicklung des Problems feststellen, das ich heute morgen unter dem Überschrift Der Jesus der Geschichte und der Christus des Glaubens ${ }^{8}$ dargestellt habe. Und die Bultmannianer haben sich, wie ausgeführt, leicht mit einem Nebeneinander zufrieden gegeben, auf jeden Fall mit einer gewissen Trennung: Es gibt einerseits das, was man aus der Geschichte über das Leben Jesu wissen kann, andererseits gibt es die Grundlegung des Glaubens der Kirche in Christus. Es gab also einen gewissen Bruch zwischen dem Jesus der Geschichte und, wenn Sie wollen, dem Christus des Glaubens. Die Mehrheit der Mitglieder der post-bultmannschen Schulen sind im Gegenzug sehr besorgt, erneut den Jesus der Geschichte zu erlangen, um im vertieften Verstehen des Jesus der Geschichte die Verwurzelung des Glaubens der Kirche zu suchen - daher ein gewisser Frühling der historischen Jesus-Forschung, der sich in der umfassenden Bibliographie der in den letzten Jahren zum Thema erschienenen Arbeiten zeigt.

Auf den ersten Blick scheint dieses Problem in Vergleich zu den genannten etwas peripher, doch steht es dennoch in Zusammenhang mit dem, was vorausging. Bultmann hat, Dilthey folgend, recht bereitwillig zwischen dem unterschieden, was man wissenschaftlich erklären und dem, was man persönlich verstehen kann. ${ }^{9}$ Man hat den Jesus der Geschichte auf die Seite des Erklärens gestellt, den Christus des Glaubens dann auf die des Verstehens. Wenn man nun aber gerade auf diese Opposition zwischen Erklären und Verstehen verzichtet - die letzten Endes wohl desaströs ist, zutiefst desaströs durch die Spielart des Irrationalismus oder Fideismus, die sie nährt -, wenn man also auf die Unterscheidungen verzichtet, muss man auch verzichten auf eine Art Nicht-Aggressions-Pakt zwischen, wenn Sie wollen, dem Theologen

\footnotetext{
${ }^{8}$ Vgl. Ricœurs Bultmann-Beitrag im selben Heft, S. 17-35; wohl anspielend auf Rudolf Bultmann, Das Verhältnis der urchristlichen Christusbotschaft zum historischen Jesus (1960), in: Exegetica.Aufsätze zur Erforschung des Neuen Testaments, ausgewählt, eingeleitet und herausgegeben von Erich Dinkler, Tübingen 1967, S. 445-469.

${ }^{9} \mathrm{Vgl}$. Wilhelm Dilthey, Ideen über eine beschreibende und zergliedernde Psychologie (1894), in: ders., Gesammelte Schriften, Bd. V, Stuttgart/Göttingen 1957, S. 144.
} 
und dem Exegeten und das Nebeneinander zwischen dem Jesus der Geschichte und dem Christus des Glaubens aufgeben.

Es handelt sich also um einen Versuch, die theologischen Probleme besser mit den historisch-kritischen $\mathrm{zu}$ verschmelzen, um eine Veränderung des Feldes in sehr kurzer Zeit (ich spreche von den vergangenen zehn Jahren), die kommende, noch in Arbeit befindliche Werke bestimmen wird. Ich habe Ebeling ausgewählt, da seine Schriften einfacher zu lesen sind als die seines Kollegen Fuchs, dem ich mich auch hätte zuwenden können. (Ernst Fuchs, der 1954 ein sehr wichtiges Buch geschrieben hat, Die Hermeneutik ${ }^{10}$, das aber sehr viel schwieriger ist). Ich habe im Gegenzug die Aufsatzsammlung Ebelings gewählt, Wort und Glaube ${ }^{11}$, La Parole et la Foi ${ }^{12}$; sie sind Teil eines Werks, das unter anderem auch ins Englische übersetzt wurde (im Verlag der englischen Studentenverbindung) ${ }^{13}$, was uns einen zweiten Zugang zu seinen Arbeiten gibt. Die Werke Ebelings und Fuchs' verlaufen abgesehen davon nicht nur parallel zueinander, sondern sind sehr solidarisch, da sie gemeinsam eine Buchreihe gegründet haben - publiziert in Tübingen, bei Mohr $-{ }^{14}$, und sie haben zwei parallele Institute, das eine in Zürich, das andere in Marburg, die sich Institute für Hermeneutik nennen. Indem ich über Ebeling spreche, treffe ich also eine Wahl, die etwas ungerecht ist. Wie dem auch sei, ich präsentiere ihn hier als Vertreter eines Forschungszweigs.

Dies also sind die Gesichtspunkte, die ich Ihnen zu Gehör bringen und zur Diskussion vorlegen möchte: zunächst die Rückwendung [le retour] zur Reformation innerhalb des hermeneutischen Problems. Ich glaube, es ist sehr wichtig zu sehen, dass es das Nachdenken über Luther, über dessen Lektüre der Bibel war, welches diese Theologen - eher Historiker als Exegeten - das hermeneutische Problem erneuern liess. Die erste Frage lautet also: Was heisst Rückwendung zum hermeneutischen Problem der Reformation? Es ist sehr auffallig, dass die Rückwendung zu Luther sowohl bei Ebeling als auch bei Fuchs wesentlich betrachtet wird als eine Rückwendung zu dessen Hermeneutik, auffällig nämlich insofern, als die Dogmengeschichte seit der zweiten Hälfte des 19. und zu Beginn dieses Jahrhunderts wenigs-

${ }^{10} \mathrm{Im}$ Orig. dt.; eigentlich »Hermeneutik«: Ernst Fuchs, Hermeneutik, Bad Cannstadt 1954.

${ }^{11}$ Im Orig. dt.; Gerhard Ebeling, Wort und Glaube [=WG], [Bd. 1], Tübingen

${ }^{3} 1967$ (1960).

${ }^{12} \mathrm{Im}$ Orig. franz.

13 Gerhard Ebeling, Word and Faith, translated by James W. Leitch, London 1963.

${ }^{14}$ Hermeneutische Untersuchungen zur Theologie, $1962 \mathrm{ff}$. 
tens in Deutschland im Bereich der Glaubensbekenntnisse durch die Restauration der christologischen und trinitarischen Aussagen geprägt war. Dies war es, was man in der Reformation in erster Linie gesehen hat: die Geschichte ihrer Glaubensbekenntnisse und also der Theologie, die sich durch die Glaubensbekenntnisse vollzieht. Ebeling dagegen denkt, dass man zu Luther dem Exegeten zurückkehren muss. Was heisst das, und welche Radikalisierung ist damit implizit gegeben?

Nach Ebeling ist die grundlegende Idee der Reformation die, das Wort an die Stelle der Ontologie zu setzen. Um das zu erklären: Der zentrale Gedanke der reformatorischen Hermeneutik, der Auslegung der Schrift, ist der, dass wir keinen Rückhalt haben in einem metaphysischen Realismus, um den Sinn selbst der Predigt weiterzuleiten, dass wir keine andere Quelle haben als den Glauben, das heisst das Verstehen des Wortes und die Bewegung vom Wort auf das Wort hin [le mouvement de la parole à la parole]. Daraus folgt, wenn Sie wollen, eine Darstellung des Problems in zwei Reihen: auf der einen Seite, was man einen grundsätzlichen Protestantismus [protestantisme fondamental] nennen kann, und auf der anderen einen grundsätzlichen Katholizismus [catholicisme fondamental]. Der grundsätzliche Protestantismus war wesenhaft, immer und grundlegend eine Kategorie des Wortes, während der grundsätzliche Katholizismus eine Ontologie ist.

Nebenbei sei bemerkt, dass wir damit keineswegs zwei Konfessionen charakterisieren, sondern - und ich glaube, Ebeling würde dem zustimmen - zwei grundlegende Richtungen, deren eine naheliegenderweise eher durch die reformatorischen Kirchen repräsentiert wird, die andere eher durch den Katholizismus. Das soll nun aber nicht heissen, dass es im Katholizismus keine Theologie des Wortes gäbe - zumal nach dem Zweiten Vatikanum - und ebensowenig keine Ontologie im Protestantismus. Im Gegenteil, die Reformation hat stets wieder eine Art Scholastik eingeführt, weil sie sich nicht traute, aus ihren eigenen Kategorien zu leben. Verstehen Sie dies also nicht als eine Beschreibung zweier Konfessionen, sondern als eine Art Polarität, die dem Christentum vielleicht wesenhaft ist.

Was sind nun die Kategorien des Wortes im Gegenzug zu diesen ontologischen Kategorien? Wir können das Problem auf vier verschiedenen Ebenen darstellen: sei es, dass wir vom Geschehen [événement] sprechen, der Offenbarung, sei es, dass wir von der Natur der Heiligen Schrift sprechen, sei es, dass wir von der Überlieferung in einem Traditionszusammenhang sprechen, sei es schliesslich, dass wir von der Gewinnung eines historischen Bewusstseins von Kirche durch diese selbst sprechen. Auf diesen vier Ebenen und anhand dieser vier Punkte werden wir die Dualität Kategorie des Wortes/Kategorie der Ontologie nachvollziehen. Was nun das Geschehen betrifft, die Offenbarung, ruht 
der Katholizismus (als solcher, um es zu wiederholen, nur Richtung, Pol des Christentums und nicht Konfession) auf realistischen Kategorien, die in den Werken der grossen Konzilien zwischen dem vierten und dem sechsten Jahrhundert sehr gut zum Ausdruck kommen; sie präsentieren die Doppelnatur Christi, göttliche und menschliche Natur, wobei die trinitarischen Aussagen sich darstellen als Aussagen, die Physik und Metaphysik, Natur und Übernatürliches, mischen.

Nun hat aber die Reformation im Grunde, so Ebeling, diese realistischen Kategorien wenn nicht unterdrückt, so wenigstens ins Wanken gebracht, indem sie einzig auf personalen Beziehungen beruht und nicht auf ontologischen Strukturen. Die Idee der Doppelnatur, der Zugehörigkeit also zu zwei Realitätssphären, einer intelligiblen und einer sensiblen - das sind platonische Kategorien, nicht aber christliche. Nur durch die Verwechslung mit der Unterscheidung zweier Welten, der unsichtbaren von der sichtbaren Welt, und der Verbindung dieser Welten in der Person Christi hat man dem Wortgeschehen [événement de la parole] eine gewisse Konsistenz gegeben; man hat es zu einer doppelseitigen Sache gemacht, halb göttlich, halb menschlich. Das ist es auch, was wir im Begriff [notion] der Heiligen Schrift finden, dem zweiten Punkt unserer Gegenüberstellung. Hier wird die katholische Auslegung immer darauf bestehen, sich zwei Geschichten zu vergegenwärtigen, eine heilige und eine profane. Die historia sacra verläuft parallel, ist verschieden von der profanen Geschichte. Alles in allem verhält es sich mit der Doppelnatur der Schrift genau so wie mit der Doppelnatur Christi, während die Reformation - indem sie die Predigt ganz auf das Zeugnis der Schrift konzentriert - keinerlei Rückhalt hat in der Unterscheidung von zwei Naturen der Texte, zwei Schriften oder zwei Geschichten. Das Wort ist keine andere Realität neben unserer eigenen. Wir werden im Übrigen gleich sehen, wie sich die Unterscheidung von Gesetz und Evangelium mit dieser Opposition zwischen wesenhaftem Katholizismus und wesenhafter Reformation decken wird.

Mit dem dritten Beispiel der Gegenüberstellung gelangen wir ins Herz des hermeneutischen Problems. Denn hier geht es darum sich $\mathrm{zu}$ fragen, wie uns dieses Wort mitgeteilt wird. Und nun verstehen wir auch besser die Opposition zwischen den realistischen Kategorien und denen des Wortes: Alle Mediationen des Katholizismus sind realistische in dem Sinn, dass es sich um die Bewahrung einer Reliquie handelt - im strengen Sinn des Wortes -, um ein primitives Geschehen: Die berühmten Reliquien in einem pejorativen Sinn des Wortes - die Knochen oder Fingerteile von Heiligen - sind sehr aussagekräftig bezüglich einer Gesamtmentalität, einer ganzen Denkökonomie, innerhalb derer wir nicht mittels einer Auslegung rückgebunden sind an ein 
Wort, sondern gleichsam eine physische Garantie der Kontinuität eines Geschehens haben, das in Gestalt der Reliquie bis zu uns reicht.

Ich glaube, dass der Begriff der Reliquie hier sehr interessant ist, weil wir es nicht mit einer Kuriosität zu tun haben, einer Absonderlichkeit des Katholizismus, sondern mit einem Anzeiger einer Mentalität, innerhalb derer die Reliquie gewissermassen die Zusammenfassung eines umfassenden Offenbarungsverständnisses darstellt. Schliesslich versteht sich die Kirche insofern selbst als Reliquie, als die apostolische Weitergabe von Kontakt zu Kontakt wie die Fortführung eines ersten Geschehens ist, das durch seine eigenen physischen Spuren bis zu uns reicht. Die Unfehlbarkeit der Kirche ist genau nach dem Modell der Doppelnatur Christi entworfen; in der Kirche gibt es einen Teil, der fehlbar ist und der profanen Geschichte angehört, und es gibt einen unfehlbaren Teil, genau so, wie es neben der physischen eine göttliche Natur Christi gibt. So, durch diese Art einer realen Fortführung eines vergangenen Geschehens, das bis in die Gegenwart fortlebt, gelangt man gewissermassen von der Doppelnatur Christi zur Doppelnatur der Kirche.

Und dies nun ist, sagt uns Ebeling, die wesenhaft nichthermeneutische Auffassung. Dies ist in dem Sinn eine nichthermeneutische Auffassung als das Wort nicht durch ein anderes Wort überliefert wird; vielmehr wird es überliefert als Sache, welche die Geschichte durchquert, ohne von dieser affiziert zu werden. Und dann kommt Luther. Wenn Luther sagt "allein durch den Glauben", sola fide, äussert er nicht etwa ein moralisches oder gar ein Lebensprinzip, sondern vielmehr ein hermeneutisches Prinzip. ${ }^{15}$ Denn das sola fide, wallein durch den Glauben", schliesst jede andere Mediation aus als die des Wortes, das in einer lebendigen Aktualität ausgelegt wird. Das sola fide bedeutet, dass das einmalige Geschehen allein gegenwärtig wird durch ein anderes, ihm homogenes Geschehen, und dieses nämlich ist ein anderes Wortgeschehen. Hier kommen wir nun zu dieser grundlegenden Beziehung: Parole-Interprétation - Wort-Auslegung ${ }^{16}$. Einzig in dieser Verbindung zwischen einem Wortgeschehen und einem anderen Wort, das dieses auslegt, ist eine Kirchengeschichte möglich, wird Geschichte geschaffen. Die Kirche des Wortes hat keine andere Sicherheit als eine ständige Exegese [éxégese permanente], das heisst eine gegenwartsbezogene Auslegung eines Wortes, das zu einem gewissen Zeitpunkt hervorgebrochen ist.

${ }^{15}$ Vgl. Gerhard Ebeling, Wort Gottes und Hermeneutik (1959), in: ders., WG I, S. 319-348, S. 321.

16 Im Orig. dt. 
Sehen Sie nun, wie der Begriff der Exegese hier nicht bloss eine Arbeit des Spezialisten meint. Zwar gibt es Menschen, die Exegese betreiben, doch ist die Kirche durch und durch Exegese, das heisst, dass sie über nichts anderes verfügt, was sie - ihre Existenz - garantieren würde, als eine Lektüre, die sie ständig hervorbringt. Und daher rührt eben der vierte Ausdruck der Dualität: die Gewinnung des Selbstbewusstseins der Kirche. Dies ist ein wenig auch die Antwort auf eine Frage, die mir heute früh gestellt wurde. Eine Kirche, die sich als katholische Kirche versteht, ist eine Kirche, die denkt, sie entgehe der Geschichte. Sie ist unwandelbar, während alles Andere sich verändert; und im Grunde führt der Dualismus der Unwandelbarkeit und der Veränderung nur dazu, die genannten Dualismen zu wiederholen - die Doppelnatur Christi, die Doppelnatur der Schrift, die Doppelnatur der Kirche. Die Kirche der Reformation hingegen ist eine Kirche, die sich durch und durch als historische wahrnimmt, denn es gibt sie nur durch die Beziehung von Wort und Auslegung.

Dies ist, was die Reformation getan hat, doch hat sich davon nicht ein vollständiges Bewusstsein gehabt, da sie sich selbst dennoch mehr oder weniger nach einem katholischen Modell verstand. Es handelte sich darum, eine Kirche zu sein wie die katholische, nur reiner, das heisst, wie Ebeling sagt, die Reformation hat sich schlicht verstanden als eine quantitative Reduktion der Offenbarungsquellen. ${ }^{17}$ Die Katholiken haben etwas zur Schrift hinzugefügt; sie haben die Tradition hinzugefügt. Wir verzichten auf die Tradition, was heisst, dass die Zahl der Quellen kleiner wird. Was die exegetische Revolution der Reformation ausmachte, war indes nicht nur eine quantitative Reduktion der Quellen, vielmehr war sie eine tiefgreifende Transformation ihres Sinn und Gebrauchs, insofern nämlich als dieses Wort selbst nicht eine Sache ist, sondern nur existiert in seiner dauernden Reaktivierung durch ein anderes Wortgeschehen. Ich zitiere aus einem Text Ebelings: „Die Kategorie des Auslegung halte ich für die einzig sachgemäße, um die theologische Frage nach dem Wesen der Kirchen- und Theologiegeschichte in Angriff zu nehmen. Denn in der Kategorie der Auslegung ist die Geschichtlichkeit der Vergegenwärtigung des Offenbarungsgeschehens strukturell erfaßt. «"18

Dies also ist der zweite Punkt, den ich nun - wie heute früh - behandeln möchte: dieser Begriff des Wortprozesses oder des Wortgeschehens [cette notion de procès de parole, ou d'événement de parole]. Sie haben also gesehen, dass es sich bei der Rückwendung zum Wesen der radi-

${ }^{17}$ Vgl. Ebeling, Wort Gottes und Hermeneutik, S. 320.

${ }_{18}$ Gerhard Ebeling, Die Bedeutung der historisch-kritischen Methode für die protestantische Theologie und Kirche (1950), in: ders., WG I, S. 1-49, S. 24f. (kursiv von Ricœur). 
kalen Unternehmung der Reformation um eine Rückwendung zum Wort handelte. Was wollen wir sagen, wenn wir sagen, dass das Wort selbst ein Geschehen oder ein Prozess ist? Die Deutschen haben einen sehr schönen Ausdruck, um dies zu sagen: Wortgeschehen, und Geschehen ${ }^{19}$ wird im Deutschen das genannt, was passiert [arrive], was geschieht [advient], jedoch nicht in Form eines plötzlichen Ausbruchs (es geht hier nicht so sehr um die Idee der Momenthaftigkeit, des Moments, der hervorbricht [surgit] und wieder verschwindet [disparaît]), sondern als etwas, das sich entfaltet [se déploie], sich entwickelt [se dévelope]. Dies ist der Grund, weshalb ich dachte, das Wort Prozess sei vielleicht das bessere. Es gäbe, denke ich, andere Weisen zu übersetzen, etwa durch das alte französische Wort échéance ${ }^{20}$ [Fälligkeit]. Wenn wir sagen, dass das Wort fällig ist, bedeutet dies, dass es gilt, eine neue Fälligkeit zu gewähren. Ich glaube, es ist dies, was den Grund von Ebelings Denken ausmacht: Das Wort ist geschehen [advenue], doch weil es Text geworden ist [devenue], geht es darum, ständig den Text wieder in das Wort zu verwandeln. Und dies ist der Prozess, der Prozess, den er ferner recht oft auch definiert als Bewegung von der Schrift zur Predigt oder vom Text zurVerkündigung. ${ }^{21}$

Es gibt das Wort nicht anders als in dieser Bewegung. Es ist nicht eine Sache, die ihren Ort in der Welt hat oder in einer anderen Welt. Es geht nicht um eine andere Welt neben unserer eigenen. Es geht um diese Bewegung des Geschehens des Wortes [advenir d'une parole], die, einmal geschehen [advenue], erneut geschehen [advenir] muss. Hier kann man, glaube ich, wirklich von einer Theologie des Wortes sprechen, und dies zugleich in einem positiven wie in einem negativen Sinn: in einem negativen Sinn, weil dies eine Theologie ist, die nichts anderes sein will als eine Theologie des Wortes, die keinerlei Vorstellung davon hat, was die Welt ist, die keine Kosmologie umfasst, keine Physik, keine Anthropologie, keine Psychologie, die über all dies nichts aussagt. Eine Theologie also, die ganz und gar ausgerichtet ist auf Gott als Wort und auf das Wort Gottes. Ich glaube, dass diese Form derVereinfachung und Radikalisierung der Theologie aber zugleich bedeutsam ist für unsere Zeit, in welcher die Theologie ihre Einflussbereiche nach und nach gänzlich verloren hat. Denn sie gibt nicht länger vor, eine Kosmologie zu bieten, noch eine Physik, noch eine Biologie und auch weder eine Psychologie noch eine Politik.

Was also ist sie? Sie ist eben Theologie des Wortes. Das ist, negativ gesprochen, eine Reduktion der Theologie auf sich selbst, doch ist es,

${ }^{19}$ Im Orig. dt.; irrtümlicherweise "wortgeschehen« bzw. "geschehen« (S. 43).

${ }^{20}$ Im Orig. franz.

${ }^{21}$ Vgl. etwa Ebeling, Wort Gottes und Hermeneutik, S. 345. 
positiv gesprochen, zugleich die Bewusstwerdung einer Ursprungssituation [situation originelle], die durch andere Aufgaben - herrührend von einer Theologie, die Wissenschaft von einem göttlichen Wesen sein wollte - gewissermassen verdeckt war. Hier jedoch handelt es sich ganz und gar nicht um eine Wissenschaft, weder um die eines Wesens, noch des Göttlichen. Wie aber kann man also eine Theologie des Wortes betreiben? Dies ist das Problem, das ich untersuchen möchte. Alle Bemühungen Ebelings (und ich sage nicht, dass es ihm gelungen ist, das Problem zu lösen, doch hat er es, wie ich meine, gut exponiert) richten sich auf dieses: Wie kann man sich darüber klar werden, was das Originale, Spezifische, Irreduzible ist am Wort Gottes, ohne je erneut eine Metaphysik zu errichten, ohne je eine zweite Welt neben der anderen zu errichten, ein Jenseits? Und daraus folgend, ohne je eine heilige Hermeneutik [herméneutique sacrée] einer profanen Hermeneutik [herméneutique profane] an die Seite zu stellen? Es gibt nur eine Hermeneutik, und sie ist ganz und gar profan. Und in ebendieser Profanität, in dieser gänzlichen Profanation des Wortes ist es, wo man, so könnte man sagen, den Akzent des Wortes Gottes wiederfinden muss, die Spezifität des Wortes Gottes.

Dies stellt sich zunächst ein wenig wie die Quadratur des Kreises dar, da wir es mit zwei Forderungen zu tun haben, die konträr zu sein scheinen: sich klar werden über das Wort Gottes, als von Gott stammendes, also anderen Ursprungs, ohne im Blick auf die Methode je einer heiligen Hermeneutik ${ }^{22}$, einen privilegierten Status einzuräumen, ohne erneut ein heiliges Buch an die Seite eines profanen zu legen, göttliche Wesen an die Seite nicht-göttlicher, also ohne je erneut einen Dualismus zu errichten. Dies ist, glaube ich, das grundlegende Problem einer Theologie des Wortes: Wie wird man sich klar über das Wort als Wort Gottes, ohne einen metaphysischen Dualismus zu erneuern?

Wie gesagt, Ebeling löst das Problem nicht, er gibt uns jedoch einige Ansätze, indem er über das Wort nachdenkt, und dies ohne zuerst $\mathrm{zu}$ fragen, ob es menschlich oder göttlich ist. Ich glaube, es ist sehr wichtig, diese Denkmethode gut zu verstehen. Lassen Sie uns darüber nachdenken, was ein Wort sein könnte, von dem wir annehmen, es sei unter uns, wie ein erfülltes Wort, ein mit den einzigen Möglichkeiten unseres Menschseins gesprochenes Wort. Nun, was also ist ein Wort? Dies ist der Punkt, an welchem Ebeling seine Analyse weiter zu treiben versucht.

Hier nun einige Elemente eines Lösungsansatzes. Zunächst muss gesagt werden:Verstehen ist nie Verstehen der Sprache, sondern Verstehen

${ }^{22}$ Im Orig.: »un statut privilégie à une hérmeneutique sainte, à une hérmeneutique sacrée« (S. 45). 
durch die Sprache. Dies ist eine erste Anmerkung, zunächst harmlos scheinend, doch birgt sie viele Konsequenzen. Die Sprache selbst ist keine Welt und auch kein Objekt, sondern stets ein Milieu, ein Medium. Das Verstehen ist nichtVerstehen von Sprache, sondern Verstehen durch Sprache ${ }^{23}$ und diese Präposition à travers ${ }^{24}-$ durch $^{25}$ - bedeutet, dass das Wort durchquert wird. Es hat seinen Sinn also immer jenseits seiner selbst. Was das Wort auszeichnet, ist, dass es sich auf etwas anderes hin überschreitet. Wenn also (und hier zitiere ich Ebeling) »der Wortprozess sachgemäss stattfindet (und noch einmal: ich frage nicht danach, ob es sich um ein menschliches oder um ein göttliches Wort handelt, ich spreche vom Wort), ist dieses Wort selbst ein Aufruf zum Verstehen. Das Verstehen ist ihm nicht äusserlich, sondern es versteht sich selbst; es überschreitet sich selbst hin auf sein eigenes Verstehen. ${ }^{26}$

Dieser Gedanke eines Selbst-Verstehens [auto-compréhension] des Wortes - wenn das Wort angemessen geschieht, bewirkt es eine Bewegung des Verstehens -, dieser Gedanke ist zugleich sehr alt und sehr modern. Und was Ebeling diesbezüglich auszeichnet, ist, glaube ich, eine Art Überlagerung von Luther und spätem Heidegger. Historisch betrachtet ist dies ein seltsames Phänomen, da man die Behauptung, dass das Wort Interpret seiner selbst ist, bei Luther sehr oft findet. Die Schrift ist Interpretin ihrer selbst. Dies hatte bei Luther einen polemischen Sinn, da er sagte, dass deshalb kein Bedarf an Priestern sei: Das Wort selbst entwickelt sein eigenes Verständnis. In exegetischer Hinsicht ist die Idee jedoch sehr tiefsinnig. Scriptura interpres sui, die Schrift ist Interpretin ihrer selbst. ${ }^{27}$ Und wenn sie Interpretin ihrer selbst ist, so heisst das, dass es der Lebenssinn ist, der sich durch den Ausdrucksakt [expression] ankündigt, das, was Luther - vielleicht etwas unglücklich - die Dinge [les choses] nennt. Doch hat er die Dinge den Zeichen entgegengesetzt, die Dinge den Wörtern [mots], wenn er sagt: "Die Dinge sind unsere Hauslehrer, man muss die Wörter den Dingen unterwerfen. $\aleph^{28}$ Die Dinge sind unsere Hauslehrer, dies sollte bedeuten: Es sind nicht die Worte, die sprechen, sondern durch die Worte wird etwas gesagt, und das Gesagte ist unser Hauslehrer. Auf diesem Weg befiehlt uns das Wort; das Wort ist unser Lehrer, weil der Sinn, der jenseits der Wörter

${ }^{23}$ Vgl. Ebeling, Wort Gottes und Hermeneutik, S. 333.

${ }^{24}$ Im Orig. franz.

${ }^{25}$ Im Orig. dt.

${ }^{26}$ Das Zitat ist bei Ebeling so nicht zu finden; zur Sache jedoch: Ebeling, Wort Gottes und Hermeneutik, S. 331-335; 340ff.

${ }^{27}$ Vgl.WA 7; 97, 23-29 (vgl. Ebeling, Wort Gottes und Hermeneutik, S. 320).

${ }^{28}$ Vgl. WAT 5; 26,11 (Nr. 5246) bzw. WA 5; 634,14-16; vgl. Ebeling, Wort Gottes und Hermeneutik, S. 336, Anm. 26. 
geschieht, die Überschreitung der Wörter befiehlt in einer endlosen Interpretationsbewegung.

Das Verstehen ist also nicht etwas, das wir hinzufügen, das wir dem Wort anheften, vielmehr ist es eine Bewegung, die vom Wort selbst bewirkt wird. Ich habe gesagt, dass das von Luther kommt, das ist wahr. Es ist Luther, der behauptet, dass die Schrift die Arbeit der Exegese hervorbringt. Der Gedanke ist aber auch dem späten Heidegger nahe, dessen wundervollen Texten über die Sprache, in denen er sagt, dass die Sprache sprich $t^{29}$. Sie ist Entfaltung ihres eigenen Sinns, und wie ich heute früh sagte: Es besteht hier die Möglichkeit einer neuen Philosophie, die sehr viel weniger subjektivistisch ist, wenn Sie wollen, sehr viel weniger existenzialistisch, innerhalb derer das Subjekt des Wortes einen sehr viel geringeren Stellenwert besitzt als diese Selbst-Manifestation des Wortes in sich selbst [auto-manifestation de la parole en elle-même]. Wir gehen einen Schritt weiter, wenn wir bedenken, dass nicht nur das Wort seinen eigenen Sinn überschreitet, dass sein Sinn eine Auslegung entfaltet, sondern dass jedes Verstehen darüber hinaus darauf abzielt, in einer Begegnung zu verschwinden oder zu sterben. Selbst dort ist das Wort wesentlich Mediation, das heisst: Die Aktualisierung eines Wortes ist immer etwas, das der Ordnung der Stille angehört, der Ordnung des Kontakts, wobei Kontakt hier völlig metaphorisch gemeint ist.

Unser eigentlicher Bezug zum Wort ist nicht, dieses in Umlauf zu bringen, sondern es zu hören [ouïr], ihm zuzuhören [écouter], und in diesem Zuhören ruft das Wort zu einer Art Erfahrung, das heisst zur Begegnung mit etwas oder jemand, der vom Wort eingesetzt wird, eine Begegnung und Kontaktnahme eingesetzt durch das Wort. Hier kann man tatsächlich sagen, dass das Wort sterben will, weil es verschwindet - zugunsten dieser Vergegenwärtigung, dieses Präsent-Machens. Dies ist, was Heidegger sehr oft zum Ausdruck gebracht hat indem er sagte, dass das Ziel des Sagens [dire] das Zeigen [montrer] sei, das Sehen-Lassen [faire voir], und das Sehen-Lassen ist ein Sein-Lassen [laisser être] vor uns. Dieser Objektivismus des Wortes wird sehr weit getrieben, nämlich bis zu einer Art Selbst-Unterdrückung des Wortes in Bezug auf das, was es sagen will. Und im Grunde finden wir diesen Gedanken auch bei Luther, wenn er uns sagt, das Proprium des Exegeten sei letztlich anzukommen bei einer Art Begegnung von Person und Person. Die Begegnung, bei Luther in personalistischen Kategorien zum Ausdruck gebracht, ist wie der Streit, in dem das Verstehen des Wortes stirbt und erlischt.

Mit diesen Elementen einer Meditation über das Wort gelingt es uns nun vielleicht besser, mit besseren Begriffen, das Problem zu formulieren, das uns zunächst eine Art Widerspruch zu sein schien: wie

29 Martin Heidegger, Unterwegs zur Sprache, Pfullingen 1979, S. 12. 
den göttlichen Ursprung eines Wortes erhalten, ohne erneut einen Dualismus realistischen Charakters aufzubauen zwischen zwei Welten, zwei Ordnungen von Realität?

In welchem Sinn können wir also vom Wort Gottes sprechen? Ebeling schlägt vor, es im Sinn derVorstellung eines erfüllten Wortes zu tun. Ein erfülltes Wort, das heisst ein Wort, das Kommunikation schafft, das Existenz schafft, das Klarheit schafft. Wir könnten sagen, dass wir in dem Masse einem Wort Gottes gegenüber stehen, in dem wir einem Wort gegenüber stehen, das ein Verstehen seiner selbst in Bewegung setzt, ein Verstehen des Fremden [autrui], das eine Geschichte hervorbringt und, folgedessen, lebendig, wirksam ist. Denn dies wäre ein Wort, das den Menschen hervorbrächte. Sie sehen, hier erreichen wir nun eine theologisch ganz und gar grundlegende Thematik.

Das einzige Kriterium aufgrund dessen wir sagen könnten, dass ein Wort von Gott stammt, läge im Mass, in dem es gänzlich für den Menschen ist. Seine Fähigkeit, Menschlichkeit hervorzubringen, ist seine Göttlichkeit. Seine Göttlichkeit ist also nichts anderes als die Eröffnung einer Geschichte der Mitteilung und also der Fülle unserer Sprache. Hier greift Ebeling ein Thema aus dem Römerbrief des Paulus auf, die Opposition des wahrhaftigen Gottes [Dieu véridique] und des verlogenen Menschen [homme menteur] (Rom 3,4). Gott ist wahrhaftig, der Mensch ist verlogen, was heissen soll, dass dies in gewissem Sinn zwei Pole innerhalb des Wortes sind. Wir haben nur ein Wort, es gibt nur eine Welt des Wortes, doch diese Welt des Wortes hat zwei Pole: Es hat einen göttlichen Pol, der seine Wahrhaftigkeit ist und sein Lebenscharakter, seine Fähigkeit, Leben hervorzubringen. Und was nur menschlich, allzumenschlich ist, um wie er zu sprechen, eben dies ist nicht wahrhaftig im Wort, ist, was Paulus "Lügner" nennt. Im Lateinischen werden verax/mendax in Opposition gebracht. ${ }^{30}$ Es ist der wahrhaftige Gott und der verlogene Mensch. Es handelt sich hier jedoch nicht um zwei Seinsbereiche, sondern um zwei Pole eines einzigen Wortes. Ich zitiere aus einem Text Ebelings: "[D]as Wort Gottes [ist] deswegen letzter Grund des Verstehens [...], weil hier in letzter Hinsicht Wort als Wort und Verstehen als Verstehen begegnet. ${ }^{31}$

Dies ist eine Auffassung des Problems, die sich etwa von jener Barths, der in seinem bekannten Buch bzw. seiner Aufsatzsammlung Wort Gottes und menschliches Wort einander gegenüberstellt, grundsätzlich unterscheidet. ${ }^{32}$ Ebeling, und in einem generellen Sinn die Post-

\footnotetext{
${ }^{30}$ Vgl. Ebeling, Wort Gottes und Hermeneutik, S. 341.

31 Ebd. S. 340 (kursiv von Ricœur); Ricœur übersetzt die Stelle recht frei.

${ }^{32}$ Vgl. Karl Barth, Das Wort Gottes und die Theologie, München 1924; die französische Übersetzung (Paris 1933) trägt den Titel Parole de Dieu et parole humaine.
} 
Bultmannianer, haben Barth vorgeworfen - obwohl er dies nicht wollte -, eine Art metaphysischen Dualismus wiedererrichtet zu haben, einen Bereich des Wortes Gottes, der dem Bereich des menschlichen Worts gegenübersteht. Nein, es ist das Mass, in dem es nur ein Wort gibt, welches voll oder leer sein kann, in welchem es eine Polarität Gott/Mensch gibt. Es gibt nicht zwei Sprachen, eine göttliche und eine menschliche, es gibt nur eine einzige: das Wort des Menschen, doch dies je nachdem, ob es gänzlich geschehen ist oder nicht, geschehen in einem so vollständigen Sinn, dass Wortsein und Menschsein eins ward. Auf diese Weise versucht Ebeling das Johannes-Wort zu verstehen: »Das Wort ist Fleisch geworden ${ }^{33}{ }^{33}$ Das will sagen: Es gab in der Geschichte einen Moment - und dies ist das christliche Kerygma -, in dem das Wort ganz erfüllt wurde. Das fleischgewordene Wort, dies ist die Möglichkeit, dass die Menschen künftig statt zu lügen ein wirksames Wort aussprechen, das die Wahrheit sagt. Wenn die Bibel vom Wort Gottes spricht, kann sie nichts anderes sagen; sie will kein anderes Wort sagen, sondern das Wort als natürliches Wort, mündlich, Wohnung nehmend unter den Menschen. Sagen, dass Gott der Ursprung ist, das bedeutet, dass er deroder dasjenige ist, von dem ein Wortprozess [procès de parole] ausgehen kann, wo das Wort ganz Wort ist, denn Sünde bedeutet gerade, dass wir diesem Wort, in dem der Sinn spricht, nicht gewachsen sind.

Ebeling zieht daraus den Schluss, dass es keinerlei Notwendigkeit gibt, eine heilige von einer profanen Geschichte [histoire sainte/profane] zu unterscheiden, eine heilige Hermeneutik von einer profanen; es gibt nicht zwei Welten des Wortes, es gibt nicht zwei Schriften, es gibt nicht zwei Naturen, es gibt nicht zwei Seinsweisen [êtres] - es gibt nur ein Wort, ein Wort jedoch, das sich, in seiner Fülle, verrät [se dénonce] als Wort Gottes, und das sich, in seiner Lügenhaftigkeit, ankündigt [s'annonce] als menschliches Wort. Ich versuche Ebelings Unternehmen $\mathrm{zu}$ verstehen, sein halsbrecherisches, schwieriges Unternehmen. Es besteht zunächst im Verzicht, sich alles zuzugestehen (ich meine die Idee eines Wortes, das anderer Art ist unser eigenes), sondern ausschliesslich zu überlegen, was ein erfülltes Wort sein könnte - um im Inneren dieserVorstellung des erfüllten Wortes die Möglichkeit einer Dualität Gott/Mensch zu finden, die gänzlich in dem enthalten ist, was man eine Art Monismus des Wortes nennen könnte.

Wir werden nun einige Folgerungen ziehen in Bezug auf das Problem, das ich zu Beginn angesprochen habe.Vielleicht werde ich dies aber besser tun, indem ich auf Ihre Fragen antworte. Ich möchte also zwei Folgerungen nachgehen. Die erste betrifft die historischkritische Methode, die Integration der kritischen Methode in die

${ }^{33}$ Joh 1,14.Vgl. Ebeling, Wort Gottes und Hermeneutik, S. 341. 
Geschichtswissenschaft, die historisch-kritische Methode in der Predigt der Kirche; die zweite betrifft die Konfrontation mit einer nicht-religiösen Welt. Ich glaube, dass diese beiden Folgerungen wichtig sind, die eine vor allem für die exegetischen Fachleute, doch: Sind wir das vielleicht jetzt nicht in verschiedenem Masse selbst, denn nebenbei gesagt: Ich sehe nicht, weshalb wir weniger intelligent sein sollten, wenn es um die Schrift als wenn es um unser Geschäft geht, unser professionelles Tun.

Wir müssen uns von einem gewissen Infantilismus des Glaubens verabschieden, und wahrscheinlich kann der Glaube eines modernen Menschen nur einer sein, der durch die kritische Bewährung hindurch gegangen ist, der sich, wenigstens im Blick auf die allgemeine Kultur, die wesentlichen Ergebnisse der modernen Bibelkritik zu eigen gemacht hat. Abgesehen davon ist dies gegenwärtig nicht sehr schwierig, denn es gibt sehr gute Arbeitsinstrumente. Ich denke insbesondere an dieVeröffentlichungen Pater Xavier Léon-Dufours über die Entstehung des Neuen Testaments. ${ }^{34}$ Man kann jetzt sagen, dass es eine Art Exegese gibt, die den öffentlichen Raum erreicht hat, die jeder Angehörige einer Kirche sich zu eigen gemacht haben sollte. Doch muss man gut verstehen, weshalb wir dies tun sollen und - vor allem - warum dies möglich ist. Und hier ist eine Theologie des Wortes nützlich, allerdings nicht um uns zu sagen, was die Ergebnisse der historisch-kritischen Methode sind, sondern um uns zu zeigen, dass die historisch-kritische Methode dem Glauben nicht fremd ist, sondern gerade Teil hat an der Bewegung des Wortes.

Bis in die jüngste Generation schien die Bibelkritik nicht nur als befremdendes, sondern oft gar als feindschaftliches Tun. Und um die Heilige Schrift gewissermassen vor den Methoden zu schützen, die auf alle anderen Schriften angewendet wurden, haben wir uns auf eine Art Fundamentalismus zurückgezogen. Nun, sagt uns Ebeling, diese Zeit ist vorbei, denn es gibt nicht zwei Weisen, Texte zu lesen, sondern nur eine: Die biblischen Texte sind Texte wie alle anderen. Die Frage ist vielmehr, ob es sich um sprechende Texte [textes parlants] handelt oder um nichtsprechende [non-parlants]. Die Unterscheidung zwischen Wort Gottes und menschlichem Wort geschieht nicht auf der Ebene der Methoden, so als gäbe es besondere Methoden für die Bibel und dann noch andere für die anderen Bücher.

In methodologischer Hinsicht gibt es keinerlei Differenz; es gibt nur eine Hermeneutik. Ich glaube, es ist nicht nur sehr befreiend, dies $\mathrm{zu}$ wissen, sondern dies theologisch zu wissen und die tiefe Einheit der Hermeneutik theologisch zu bedenken. Nun können wir verstehen,

${ }^{34}$ U. a. Etudes d'Evangile, Paris 1965 (Parole de Dieu, 2). 
dass der Geist der historisch-kritischen Methode, der oft ein Geist des Hochmuts und der Revolte zu sein schien, grundsätzlich ein Geist der Willigkeit zum Sinn war, ein Wille, die Texte zu verstehen und die Bibel auf die gleiche Weise zu verstehen, durch die gleiche Arbeit, mit der gleichen Bemühung, der gleichen intellektuellen Redlichkeit, mit welcher man versucht hat, die Ilias und die Odyssee und alle anderen literarischen Texte zu verstehen. ${ }^{35}$ Denn die Redlichkeit, von der Nietzsche gesprochen hat, diese intellektuelle Tugend schlechthin, hat an jedem Wortprozess, jeder Aktualisierung des Worts, Anteil.

Wie soll man auch ein Wort zum Leben erwecken, wenn wir es von vornherein als toten Buchstaben behandeln, geschützt gerade vor der kritischen, der verstehenden Intelligenz? Dies ist, weshalb wir es nicht tun können. Zweitens handelt es sich nicht nur darum, nicht zwei Hermeneutiken einander gegenüberzustellen, sondern auch nicht Erklären und Verstehen. Hier hat Ebeling, glaube ich, gut daran getan, diese trügerische Opposition aufzuweichen, die wir seit Dilthey mitschleppen. Diese Opposition zwischen Erklären in den Natur- und Verstehen in den Geisteswissenschaften, diese Opposition ist ausserordentlich gefährlich, weil sie zugunsten eines methodischen Dualismus letzten Endes alle anderen Dualismen wiederherstellt. Im Gegensatz dazu muss man sich sicher sein, dass man etwas zu verstehen gibt, indem man es immer besser erklärt, und dass verstehen nichts anderes bedeutet, als die Aneignung einer vollständigen Erklärung.

Sie sehen nun die Beziehung zu unseren Anfangsbemerkungen über - ein weiterer Dualismus - den Jesus der Geschichte und den Christus des Glaubens. Nun, auch dieser Dualismus muss abtreten, denn wenn wir die kritische Arbeit weit genug treiben, bringen wir diese Schrift zum sprechen. Ebeling hat darüber, über das, was er transformierende Interpretation [interprétation transformante] nennt, sehr schöne Passagen geschrieben. ${ }^{36}$ Er hält fest, dass es das Ergründen der historische Arbeit ist, welches die Texte zum Sprechen bringt, und dass das Verstehen am Ende des Erklärens erfolgt und nicht neben, oberhalb oder ihm gegenüber. Also darf man Kritik und historische Aktualisierung einander nicht entgegensetzen, denn das war stets ein wenig die Tendenz in einem modernen, postkritischen Fideismus. Hier also eine erste, ganz grundlegende Applikation. Das reformatorische Christentum ist ein Christentum, das im Stande ist, die historisch-kritische Methode zu akzeptieren, und auf diesem Weg finden wir die tiefe Einheit von Reformation und Renaissance wieder: die Reformation,

\footnotetext{
${ }^{35}$ Vgl. Gerhard Ebeling, Die »nicht-religiöse Interpretation biblischer Begriffe» (1955), in:WG I, S. 90-160, S. 104ff.

${ }^{36}$ Vgl. etwa Ebeling, Die Bedeutung der historisch-kritischen Methode, S. 36.
} 
die auf das sola fide zurückgekommen ist, und die Renaissance, die auf die wissenschaftliche Redlichkeit gegenüber den Texten zurückgekommen ist. Das sola fide und die wissenschaftliche Redlichkeit sind ein und dasselbe, und ihre tiefe Einheit gilt es zu verstehen. Denn es ist dieser Weg, der uns erlaubt, moderne Christen zu sein, das heisst: Menschen der Kritik. Nun, lassen Sie uns dies ganz und gar sein, und lassen Sie uns gläubige Menschen sein in und durch die historische Bewährung.

Ebeling - und ich weiss nicht, ob er darin recht hat - praktiziert eine Exegese der "Zeitgemässheit [conformément au temps]. Er sagt, stets sei geschrieben "und dies wird geschehen gemäss der Zeit $*{ }^{37}$ Die Zeitgemässheit für uns, heute, die zeitgemässe Predigt, ist eineVerkündigung im wissenschaftlichen Geist und also gemäss der historisch-kritischen Methode. Zweite Konsequenz: die Konfrontation mit und, bis zu einem gewissen Grad, die Übernahme der Kategorien einer nicht-religiösen Welt. Dies ist die Bonhoeffersche Seite bei Ebeling, die man auch bei einigen anderen deutschen oder auch us.-amerikanischen Theologen wiederfindet. Sie gehen bis zu dem Punkt, wo die Theologie im Grunde Christologie wird, und zwar nichts als Christologie, wo wir von Gott also nur reden können als vom Gott Jesu Christi. Der vollständige Verzicht auf den Gott der Philosophen, und schliesslich auch auf den der dogmatischen Theologie, muss die letzte Konsequenz dieser Theologie des Wortes sein, Theologie des Wortes, die uns je nur Jesus Christus begegnen lässt und dem Gott Jesu Christi, jedoch nie dem Uhrmacher-Gott, dem Gott der absoluten Intelligibilität.

Der Atheismus, sofern er Liquidation des Gottes der Philosphen ist, hat folglich Anteil an dieser Theologie des Wortes. Hier ergibt sich die Möglichkeit eines - ich würde nicht sagen: christlichen Atheismus, sondern einer atheistischen Dimension des Christentums, die zu bedenken uns im Grund bereits Barth gelehrt hat, wenn er sagt, dass das Christentum keine Religion, keine Theorie eines göttlichen Wesens sei, sondern allein des Wortgeschehens. ${ }^{38}$ Die nicht-religiöse Interpretation, von der Bonhoeffer spricht, geht folglich ebenso direkt aus dem Glauben an Jesus Christus hervor wie aus dem, was er auch die Existenz für die Anderen genannt hat. ${ }^{39}$ Ebeling wird hier sehr deutlich: Die nicht-religiöse Interpretation und die christologische Interpretation fallen in eins. ${ }^{40}$ Sie sehen, es gibt hier eine Öffnung hin auf ein pastoral-kirchliches Problemfeld, das wir im Moment erst im Begriff sind zu entdecken. Wer ist das eigentliche Gegenüber der Predigt? Ist es ein religiöser

\footnotetext{
${ }^{37}$ Vgl. ebd., S. $11 \mathrm{ff}$.

${ }^{38} \mathrm{Vgl}$. Karl Barth, KD I/2, § 17.

${ }^{39}$ Vgl. Ebeling, Die »nicht-religiöse Interpretation«, S. 100; 160.

${ }^{40}$ Vgl. ebd., S. 100.
} 
Mensch, wie dies bis jetzt immer der Fall war, da es während der 2000 Jahre des Christentums immer eine religiöse Voraussetzung gab? War es ein Unfall in der Geschichte des Christentums, dass es sich an eine religiöse Periode der Menschheit gebunden vorfand?

Kann man sich das vorstellen, eine nicht-religiöse Periode der Menschheit, und entsprechend eine Predigt, die überhaupt nicht mehr die Voraussetzung eines religiösen Menschen machte, eines Menschen, der bereits Ideen hat über Gott und über eine Art fromme Innerlichkeit ${ }^{41}$, ein Mensch - folgedessen - ohne Frömmigkeit, der in seiner gewissermassen nackten Menschlichkeit vor einem Wort stünde, das ihm keinerlei heilige Kosmologie geben würde, keinerleiVision der Welt, sondern allein die Anzeige eines Geschehens, aufgrund dessen er sprechen und leben könnte?

Dies also die Möglichkeit, die uns Ebeling eröffnet indem er Bonhoeffer folgt, dessen Impuls jedoch ganz in seine eigene Theologie des Wortes aufnimmt. Ich will damit sagen, dass das Auftreten des nichtreligiösen Menschen nicht nur nichts Skandalöses und Unerwartetes, sondern in gewisser Weise dem Grundzug, der Leitlinie der christlichen Offenbarung gemäss wäre. Hier versucht Bonhoeffer (und dies ist der Punkt, an dem er Bultmann vielleicht am nächsten steht) die Situation des Menschen eines nicht-religiösen Christentums mit ganz lutherischen Kategorien zu denken, eben etwa der Opposition von Glaube und Gesetz.

Ebeling hat sich bemüht, diese neue kulturelle Situation durch einen Rückgriff auf das lutherische - und also paulinische - Thema zu bedenken, dass das Gesetz, wie Luther sagt, die Negation Christi ist, wobei das Gesetz kulturell heute die Ordnung der Religiosität bedeute, unter welcher der westliche Mensch bis jetzt gelebt habe. Wenn es wahr ist, dass das Gesetz die Negation Christi ist, so ist es wahr, dass die Opposition Gesetz/Evangelium grundlegend ist. Wir müssen versuchen, die Beziehung von Religion und Glaube zu verstehen, und auch den Gegensatz von Religion und Glaube, indem wir uns an diesem zentralen Gegensatz des Evangeliums orientieren, dem Gegensatz Gesetz/Evangelium. Ich lese noch einen Text Ebelings, den letzten, den ich Ihnen vorlegen werde: "Nicht-religiöse Interpretation meint Gesetz und Evangelium unterscheidende Interpretation. ${ }^{42} \mathrm{Und}$ damit will ich nicht sagen, wir wüssten, was der Gegensatz von Gesetz und Evangelium sei; jede Epoche muss ihn aufs Neue auslegen. Für uns bedeutet der Gegensatz Gesetz/Evangelium den Gegensatz Religion/Glaube, was heissen soll,

${ }^{41}$ Im Orig. irrtümlicherweise "infériorité « statt "intériorité (S. 52).

42 Ebeling, Die »nicht-religiöse Interpretation", S. 139 (kursiv von Ricœur). Die Thematik wird auf den Seiten 139-154 ausführlich entfaltet. 
dass wir nicht von vornherein wissen, was der Gegensatz Gesetz und Evangelium meint. Doch gerade indem wir diesen Gegensatz auf den Gegensatz Glaube/Religion anwenden, erneuern wir den Sinn des Gegensatzes Gesetz/Evangelium.

Nun wage ich zu sagen - und mit dieser appetitanregenden Folgerung will ich schliessen: Ebeling bietet eine immense Aufgabe, innerhalb derer man Gott gänzlich neu denken müsste, nämlich als nichts anderes als das von Gott [de Dieu] in der Wendung Wort von Gott [parole de Dieu], und den Menschen als den, der dem Wort begegnet, der nur durch das Wort lebt. Dies würde heissen, dass alle unsere Vorstellungen von der Realität und vom Sein angepasst werden müssten an eine Theologie des Wortes. 